\title{
Noticias de sucesos como fuente de inspiración para la escritura creativa en E/LE
}

\author{
DOROTA KOTWICA \\ Universidad Pedagógica de Cracovia \\ dorota.kotwica@up.krakow.pl
}

\begin{abstract}
Resumen: La presente experiencia práctica es una propuesta de la explotación de noticias de sucesos para actividades de escritura creativa en E/LE. Tomaremos como ejemplo una noticia publicada originalmente en la sección de sucesos del periódico Las Provincias (versión digital). Explicaremos el potencial que posee este tipo de materiales auténticos para diseñar actividades de comprensión y expresión escrita (niveles B1-B2). Creemos que la mayor ventaja que ofrece el uso de las noticias de sucesos en clase-siempre que sean textos seleccionados cuidadosamente por el profesor- es que motivan y despiertan el interés de los alumnos a la misma vez que los trasladan al contexto de la actualidad española.
\end{abstract}

Palabras clave: escritura creativa, didáctica de E/LE, prensa digital, noticias de sucesos

\section{News articles as sources of inspiration for creative writing in Spanish}

Abstract: In this paper, we propose a set of activities of creative writing in Spanish that exploit a news article. We take as an example a piece of news published originally in a current-events section of the digital version of Las Provincias. We explain the potential that such authentic materials offer for designing reading and writing activities (levels B1-B2). We believe that the principal advantage of using news articles in class (if adequately selected by the teacher) is that they motivate and inspire the students as they transfer them to the context of the Spanish current events.

Keywords: creative writing, Spanish as a second language, digital press, news article

\section{Introducción. Textos de prensa en la didáctica de E/LE}

La prensa (también en su versión digital) se presenta como una fuente de material auténtico muy valioso para la enseñanza de idiomas (Cassany 2006; Pinilla Gómez 2006). De acuerdo con Cassany (2006: 96), el uso de la prensa en la didáctica de lenguas ofrece numerosas ventajas. A continuación, reproducimos la lista de las mismas que señala el citado autor:

- introduce la realidad de la comunidad en el aula del centro escolar;

- integra la escritura con otros códigos corrientes (fotografía, infografía, esquemas);

- incrementa el conocimiento cultural del aprendiz;

- utiliza escritos auténticos - que no fueron «preparados para la enseñanza»- y el idioma de la calle, el que usan los hablantes en el día a día;

- permite trabajar los discursos y la terminología de varios campos del saber humano, puesto que los periódicos incorporan secciones de sociedad, política, ciencia, meteorología, economía, y

- favorece el aprendizaje interdisciplinario, puesto que cada noticia o reportaje suele recoger datos, reflexiones o consecuencias que pertenecen a diferentes disciplinas. 
La anterior lista coincide, en buena medida, con las ventajas del empleo de la prensa digital en el aula que señala Pinilla Gómez (2006: 491-492). Esta autora destaca la variedad de contenidos que brinda la prensa digital, la autenticidad de los materiales que ofrece, su fácil accesibilidad (incluida la accesibilidad a las diferentes variedades diatópicas del español), la velocidad con la que se transmite la información, la hipercontextualización, la familiaridad previa de los alumnos con este medio de transmisión de la información y la posibilidad de personalizar los contenidos a los que se accede.

Se concluye, pues, que muestras de prensa digital destacan por su carácter auténtico y porque dan la posibilidad de introducir en el aula los contenidos culturales y relacionados con distintos ámbitos de la vida y del saber humano. Además, no se puede obviar la cuestión de su accesibilidad ya mencionada. En este sentido, García Mata (2003: 886887 ) destaca la adecuación de los materiales (especialmente audiovisuales) procedentes de Internet como portadores de autenticidad al aula, pues son «fáciles de almacenar y reproducir, combinan estímulos visuales y auditivos facilitando de una manera natural la comprensión, ofrecen información real no adaptada, y eliminan las barreras geográficas siendo accesibles tanto en el contexto de aprendizaje de segunda lengua como de lengua extranjera» (García Mata 2003: 887).

Estos últimos puntos, la eliminación de barreras geográficas y la accesibilidad de los materiales publicados en la prensa digital parecen bastante relevantes desde el punto de vista práctico. La posibilidad de servirse de los textos periodísticos publicados en línea es, sin duda, muy apreciable desde la perspectiva de un profesor de E/LE residente en el extranjero que no tiene acceso diario a otro tipo de materiales auténticos.

Finalmente, cabe mencionar que también en el Plan Curricular del Instituto Cervantes (PCIC) se hace alusión a diversos géneros periodísticos. Los encontramos dentro del apartado de «Géneros discursivos y productos textuales». En el «Inventario» de PCIC dedicado a este tipo de conocimientos, los textos periodísticos aparecen a partir del nivel A2, y como primera muestra de los mismos, las noticias escritas de periódicos y revistas. A partir del nivel siguiente, B1, el inventario de géneros y productos textuales que deberían conocer los aprendices aumenta sucesivamente. Aumenta, asimismo, el grado de complejidad de los textos (orales y escritos) y, aparte de géneros informativos, aparecen géneros de opinión (editoriales, reseñas, etc.).

\section{Las noticias de sucesos en el aula}

En nuestro caso, sugerimos el uso de las noticias de sucesos, un género en el que, típicamente, se relatan delitos o accidentes. Se trata de género informativo caracterizado por cierta «ligereza» de formato, pues suelen ser textos cortos y concisos, y por tanto, fáciles de comprender incluso para los alumnos extranjeros con niveles más bajos del conocimiento de la lengua. Suelen tratar temas de actualidad, si bien su trascendencia no siempre puede debatirse en términos de nacional o internacional. A menudo, especialmente en los diarios de ámbito más local, la relevancia de las noticias de sucesos se encuentra limitada a un pueblo o una provincia concreta. Las noticias de sucesos, aunque a menudo relatan accidentes, sucesos violentos y crímenes, también pueden contar hechos anecdóticos, curiosos o escalofriantes. El tipo de contenido que transmiten llama la atención y atrae a buena parte del público de la prensa, bien escrita, bien digital. Es un hecho en que reparan incluso los propios especialistas en periodismo. Con respecto 
a ello, citamos un fragmento de la noticia que resume los debates del XI Congreso de Periodismo Digital. El director de Interviú, Manuel Marlasca, reflexiona acerca de la popularidad de las noticias de sucesos y constata que ciertas noticias de sucesos, aunque aparentemente poco relevantes, pueden atraer al público más que las noticias acerca de los acontecimientos con mucha más trascendencia.

\begin{abstract}
Marlasca ha indicado que los sucesos «no son una especie en extinción», algo en lo que han coincidido sus compañeros de mesa. «El pasado 1 de marzo estuve estudiando los medios digitales más importantes y observé las noticias más leídas. Ante acontecimientos de ese día como la caída de la última cúpula de ETA o el terremoto de Chile, la más leída fue que un perro había muerto estrangulado por su propio abrigo en Madrid», ha relatado. «No es un género que desaparezca, porque cuando uno va a un bar suele hablar de un suceso, no habla de la última propuesta del Gobierno» (Aragón Digital, 2010) ${ }^{1}$
\end{abstract}

Si bien a menudo se trata de noticias que relatan hechos violentos y situaciones que atraen por su morbosidad, creemos que una noticia seleccionada adecuadamente (para el nivel de los estudiantes, su edad y madurez), permite introducir en el aula algunos temas pertinentes de la actualidad española. Con el apoyo de ciertas noticias de sucesos, se pueden comentar en clase de manera contextualizada cuestiones como los horarios de los españoles, las costumbres, la organización de la vida social, la arquitectura urbana, etc. Dependiendo de los contenidos que quiera trabajar el profesor, las noticias de sucesos pueden incluso ser el punto de partida para un debate acerca de los problemas más graves con los que lidia la sociedad actual, como por ejemplo, la violencia de género. Finalmente, queremos puntualizar que a la hora de seleccionar la noticia (o una cadena de noticias) con la que se trabajará en el aula, el profesor debe estar especialmente atento a los posibles contenidos delicados o viscerales que pueda tener. Si bien no creemos que deban descartarse por completo las noticias que traten sobre crímenes (podrían constituir un buen inicio para escritura de algún género de terror), opinamos que el tema seleccionado no debe herir la sensibilidad de los alumnos.

Enseñar los contenidos culturales y la actualidad española no son los objetivos principales de la secuencia didáctica que se presentará a continuación. Sin embargo, estos están presentes en nuestra propuesta y se introducen de manera natural, al leer, comentar e interpretar el texto de la noticia. Las actividades descritas tampoco se centran en el análisis o la escritura de géneros periodísticos. Más bien, las noticias de sucesos son, para nosotros, un punto de partida, un texto auténtico y contextualizado, con el que buscamos aumentar el interés y la motivación de los alumnos. A partir del mismo, se realizarán diversas actividades más o menos ancladas en el texto de la noticia que de manera gradual preparan a los alumnos a escribir, como parte final de la secuencia, un texto propio y original.

\title{
3. La secuencia didáctica
}

A continuación, se presenta nuestra propuesta de una secuencia de actividades en la que se parte de una noticia de sucesos. Si bien la tarea final a la que pretendemos llegar es un ejercicio de escritura con cierto grado de creatividad, se verá que, a lo largo del desarrollo

\footnotetext{
${ }^{1}$ Recuperado el 28 de mayo de 2018 de: http://www.elperiodicodearagon.com/noticias/aragon/expertosperiodismo-sucesos-aseguran-genero-no-desaparece-si-profesionales_565781.html
} 
de la secuencia, se proponen actividades de comprensión/expresión oral y lectora. Asimismo, como se ha apuntado anteriormente, se pretende que en la secuencia esté presente el componente cultural, en concreto, algunas cuestiones relacionadas con la vida diaria en España.

Creemos conveniente que, antes de comenzar las actividades centradas propiamente en una o varias noticias seleccionadas, se introduzca la definición del género de la noticia de sucesos. En vez de ofrecer una definición cerrada, proponemos realizar un pequeño ejercicio de deducción en el que los alumnos tratarán de definir la «noticia de sucesos» a partir de varios títulos de dichas noticias que se les mostrarán. Si se dispone de los medios adecuados (ordenador con conexión a Internet y proyector), sería suficiente con abrir en el navegador página de la sección de «Sucesos» de algún periódico español. Si no fuera posible, se pueden ofrecer los títulos extraídos de algún diario en línea, escritos en papel. Una vez que se haya aclarado qué es una noticia de sucesos y a qué tipo de escrito se van a enfrentar los alumnos, podemos comenzar con las siguientes actividades. Para este artículo, vamos a basar la descripción de cada actividad en una noticia concreta, que se adjunta en el Anexo 1. Sin embargo, los ejercicios que proponemos pueden repetirse y adaptarse tantas veces como se quiera con el uso de otras noticias seleccionadas por el profesor.

\subsection{Actividades previas a la lectura. Todo lo que dice el título}

Antes de realizar la lectura de la noticia, es conveniente contextualizarla y preparar a los alumnos. La relevancia del comentario previo a la lectura ha sido puesta en relieve por Cassany (2006: 71-72) quien señala que en raras ocasiones en la vida real nos acercamos a un escrito sin tener ninguna información sobre él. La contextualización de la noticia que se va a trabajar en el aula resulta, asimismo, necesaria, para «reproducir de alguna manera las condiciones reales e idóneas de lectura» (Cassany 2006: 72) y para despertar el interés de los alumnos hacia el texto. Para preparar a los alumnos para leer la noticia, proponemos las siguientes actividades:

- buscar en el mapa la localidad en la que tuvo lugar el suceso relatado en la noticia,

- buscar en internet fotos de la localidad (o incluso el barrio concreto, si se especifica en la noticia) y pedir que los alumnos compartan sus impresiones acerca de este sitio (¿parece un lugar tranquilo/peligroso/rico/pobre?, ¿con qué tipo de sucesos podría estar relacionado?),

- consultar la fecha de publicación de la noticia y reflexionar sobre la época del año en que tuvo lugar (¿qué tiempo hacía?, ¿coincidió con alguna fecha señalada?, ¿tuvo lugar en vacaciones o en medio del año escolar?),

- comentar el título de la noticia. Los alumnos intentan desambiguar aquellas partes del título que podrían entenderse de varias maneras,

- hacer predicciones sobre el tema de la noticia a partir del título.

Mostraremos la relevancia de algunos de los puntos anteriores en relación con la noticia adjuntada en el Anexo 1 y titulada «Rescatado un niño de 5 años que jugaba solo y semidesnudo en la ventana de un piso en Talavera». 
En el caso de esta noticia, resulta interesante observar, por ejemplo, la fecha de la publicación, el 26 de septiembre. Teniendo en cuenta la ubicación de la localidad en la que tuvieron lugar los hechos relatados, se puede suponer que, a finales de septiembre, no hacía ni mucho calor ni mucho frío, por lo cual el hecho de que el niño estuviera «semidesnudo» mientras jugaba solo en la ventana no parece agravar la situación.

En la lista de actividades previas a la lectura hemos mencionado, asimismo, la de descifrar las partes ambiguas del título. En el caso de la noticia anexada podríamos plantear las siguientes cuestiones:

- ¿Qué significa que el niño estaba «semidesnudo»? ¿Qué prendas llevaba puestas?

- ¿Qué significa que el niño «jugaba en la ventana»? ¿Estaba dentro o fuera de su casa? ¿Cómo son las ventanas en los pisos típicos de esta localidad? ¿Es relevante la altura del piso en el que se encontraba el niño? ¿Qué tipo de juegos podrían hacerse «en una ventana»?

- ¿Qué significa «rescatar»? ¿Quiénes son típicamente las personas responsables de un rescate? ¿El suceso tuvo un final feliz?

Se comprueba que, gracias a la realización de estas pequeñas actividades introductorias, se despierta el interés de los alumnos acerca del texto. Además, si los alumnos tienen la oportunidad de realizar las hipótesis previas sobre el artículo con la total libertad, se pueden esperar respuestas muy variadas y creativas, por ejemplo, que el niño fue rescatado por un superhéroe.

\subsection{Actividades posteriores a la lectura}

\subsubsection{Reconstrucción de los hechos}

En un segundo paso de esta secuencia didáctica, los alumnos leerán la noticia. Si es necesario, se explica el vocabulario que los alumnos no conozcan, sin embargo, se hace hincapié en que con una comprensión global de la noticia es suficiente (por ahora). A continuación, nos vamos a fijar en dos aspectos de la noticia: sus protagonistas y la cronología de los hechos presentados.

En primer lugar, se pide que los alumnos elaboren una lista de todas las personas que aparecen en la noticia, por poca que sea su aparente relevancia para el desarrollo de la acción. Por ejemplo, si trabajamos con la noticia anexada, los alumnos tendrían que mencionar a las siguientes personas: el niño, el padre, la madre, los bomberos, los vecinos, los servicios médicos.

En segundo lugar, los alumnos deben organizar, de manera cronológica, todo lo ocurrido. Para empezar, pedimos que solo hagan referencia a los hechos que se cuentan de manera explícita en el texto. La lista resultante podría tener el siguiente aspecto:

1. El padre sale de casa y deja al niño solo.

2. El niño sale a jugar a la ventana.

3. Los vecinos ven al niño y llaman a los servicios de emergencia.

4. Llegan los bomberos y rescatan al niño.

5. Los servicios médicos examinan al niño.

6. Vuelve el padre. 
7. El padre es llevado al juzgado.

8. La madre se queda con la custodia del niño.

La correcta preparación de la lista demuestra si los alumnos han comprendido el texto, y requiere que asignen los referentes a los elementos deícticos, interpreten el uso de los tiempos verbales, etc.

En un siguiente paso, los alumnos reflexionan acerca de esta primera versión de la lista y la completan con otra información que consideren relevante para comprender bien todo lo ocurrido (aunque no se haya explicitado en la noticia). Para ayudarles en esta tarea, se pueden plantear preguntas relacionadas con cada punto, por ejemplo: ¿Por qué salió de casa el padre?, ¿por qué el niño salió a la ventana?, ¿los vecinos hablaron con el niño?, ¿cómo reaccionó el niño cuando vio a los bomberos?, etc.

Como se ha visto, en esta última parte de la actividad, se pide que los alumnos vayan reconstruyendo partes de la historia que no se encuentran relatadas en la noticia. Si saben que cuentan con la libertad plena para añadir la información que falta, sus respuestas pueden ser muy variadas y muy creativas. De esta manera se preparan también para las siguientes actividades en las que se les pedirá no solo que escriban fragmentos de textos más largos (hasta ahora han escrito frases sueltas), sino que también será necesario un grado más de creatividad por su parte.

\subsubsection{Los puntos de vista}

Como siguiente paso, los alumnos elaboran pequeños textos de diferentes géneros en los que relatan lo ocurrido en la noticia desde diferentes puntos de vista: de las personas que aparecen en la misma (excluyendo, por ahora, al personaje principal, esto es, el niño). El formato del texto que se puede pedir puede ser muy variable, dependiendo del nivel del conocimiento del idioma y de los contenidos que se habían trabajado previamente. A continuación, hacemos algunas sugerencias.

- Escribe un mensaje desde el punto de vista de alguno de los vecinos que presenciaron el rescate. ¿A quién manda un mensaje para contarle el suceso que ha visto? ¿Cómo expresa su sorpresa, preocupación o indignación?

- ¿Qué dijo el padre cuando llegó a casa y vio a los bomberos y a los servicios médicos atendiendo a su hijo? Escribe una carta en su nombre en la que expresa su arrepentimiento y se disculpa por haber salido de casa dejando a su hijo desatendido.

- ¿Cómo viven los bomberos este tipo de situaciones? Escribe una nota que deja uno de ellos en su diario acerca de este día. ¿Qué pensó al recibir el aviso? ¿Cómo transcurrió el rescate? ¿Qué reflexiones tiene acerca de la actitud del padre?

Dependiendo del punto de vista elegido y el tipo de escrito que se pida, se pueden repasar antes algunas cuestiones del formato del género elegido, el grado de formalidad, etc. Asimismo, se pueden ofrecer ejemplos de expresiones y construcciones que se necesitarán para expresar las sensaciones y emociones de las que se hablará (de sorpresa, de indignación, de arrepentimiento, etc.). Las actividades de escritura propuestas en este punto pueden realizarse por parejas. Conviene ofrecerles a los alumnos un periodo de 
tiempo limitado y no muy largo para que no pierdan la motivación antes de la última tarea en la que trabajarán de manera individual y tendrán que escribir un texto más extenso.

\subsubsection{Actividad final: ¿qué pensó el niño?}

Un niño de cinco años es el personaje central de la noticia que hemos seleccionado para ilustrar la realización de esta secuencia de actividades. Sabemos qué cosas le han pasado al niño, pero no sabemos cómo el mismo vivió todo aquel día tan ajetreado. Como tarea final de la secuencia, proponemos que los alumnos relaten, en primera persona, lo que vivió el niño. ¿Qué sensaciones experimentó? ¿Se lo pasó bien jugando en la ventana? ¿Tuvo miedo? ¿Recordará aquel día durante el resto de su vida? Los alumnos contarán lo que pasó con una dificultad añadida de tener que imitar, en cierta medida, la manera de hablar de un niño. De todas maneras, de acuerdo con nuestra experiencia, los alumnos de niveles B1-B2 no tienen problemas para realizar esta actividad y la emprenden con motivación. Aunque el punto de partida de todos sea la misma noticia, cada respuesta es diferente. Puesto que a lo largo de las actividades anteriores ya habían podido reflexionar sobre lo ocurrido desde diversas ópticas, se sienten más seguros a la hora de contar su versión («infantil») de lo sucedido; también manejan ya el vocabulario necesario para narrar el suceso. Se puede pedir también que pongan títulos a sus escritos, para luego contrastarlos con el titular oficial de la noticia.

A modo de conclusión y para finalizar la secuencia, los alumnos pueden leer sus textos en pequeños grupos, corregir entre todos, compartir sus expresiones y seleccionar el mejor titular de los que se han ofrecido.

\section{Conclusiones}

En este artículo, hemos presentado una secuencia de actividades que parten de un texto breve: una noticia de sucesos. Se trata de un input de fácil acceso y que permite aportar a las actividades autenticidad, puesto que se trata de noticias que relatan sucesos reales, cuyos protagonistas son personas corrientes y que están anclados en el contexto de la actualidad española. Creemos que, gracias a estas características, las noticias de sucesos captan fácilmente el interés de los alumnos y los motivan para realizar las actividades de lectura y escritura propuestas. Además, de acuerdo con lo que indican Cassany (2006) y Pinilla Gómez (2006), hemos visto que las noticias de prensa ayudan a contextualizar el aprendizaje, aportando información sobre la realidad de la comunidad del habla española. Son, sin duda, también materiales valiosos para desarrollar el aprendizaje interdisciplinario, pues además de favorecer diversas competencias lingüísticas, invitan a ampliar el conocimiento acerca de la geografía, el clima, la cultura e, incluso, la ley española.

La secuencia que se ha presentado en este trabajo se basa en una noticia concreta, sin embargo, todas las actividades propuestas pueden adaptarse a otras noticias, dependiendo de los contenidos que quiera trabajar el profesor y de las características del grupo de aprendices. 


\section{Bibliografía}

CASSANY, D. (2006). Taller de textos. Leer, escribir y comentar en el aula. Barcelona: Paidós.

GARCÍA MATA, J. (2003). «La autenticidad de los materiales de enseñanza-aprendizaje y el uso de los medios de comunicación audiovisuales en la clase de E.L.E.». Actas del XIV Congreso Internacional de ASELE. Burgos: ASELE. Recuperado el 15 de mayo de 2018, de: https://cvc.cervantes.es/ensenanza/biblioteca_ele/asele/pdf/14/14_0883.pdf 882-894

Pinilla GómEZ, R. (2006). «Las posibilidades del periodismo digital como fuente de material didáctico para el aula de ELE». S. Pastor Cesteros y S. Roca Marín (eds.), Actas del XVIII Congreso Internacional ASELE. La evaluación en el aprendizaje y la enseñanza del español como lengua extranjera / segunda lengua. Alicante: ASELE. Recuperado el 15 de mayo de 2018, de: http://cvc.cervantes.es/ensenanza/biblioteca_ele/asele/pdf/18/18_0489.pdf

Instituto Cervantes (2006). Plan Curricular del Instituto Cervantes. Madrid: Editorial Biblioteca Nueva. Recuperado el 15 de mayo de 2018 de: http://cvc.cervantes.es/ensenanza/biblioteca_ele/plan_curricular/

ARAGÓN DigitAL (11.03.2010). «Expertos del periodismo de sucesos aseguran que el género no desaparece, pero sí los profesionales». Recuperado el 28 de mayo de 2018 de: http://www.elperiodicodearagon.com/noticias/aragon/expertos-periodismo-sucesosaseguran-genero-no-desaparece-si-profesionales_565781.html 
Anexo 1. Noticia

\section{Rescatado un niño de 5 años que jugaba solo y semidesnudo en la ventana de un piso en Talavera}

\section{J.V. MUÑOZ-LACUNA}

Toledo

Martes, 26 septiembre 2017, 14:43

Las Provincias $^{2}$

Un hombre ha sido detenido en Talavera de la Reina (Toledo) como presunto autor de un delito de abandono temporal de un menor después de que dejara a su hijo de 5 años solo en su domicilio. El menor acabó siendo rescatado por los bomberos cuando jugaba en el alféizar de una ventana.

Los hechos ocurrieron en la noche del pasado lunes cuando varios vecinos que caminaban por la calle de Aragón de esta localidad se percataron de la presencia de un niño de corta edad jugando en el voladizo de una ventana de un primer piso y alertaron a los servicios de emergencia.

Cuando efectivos del Parque de Bomberos de Talavera y agentes de la Policía Nacional se personaron en el piso hallaron al niño semidesnudo. Minutos más tarde acudieron los servicios médicos para examinar al menor, momento en el que apareció el padre, que fue detenido por un presunto delito de abandono temporal de un menor que el Código Penal castiga con pena de prisión de 18 meses a 4 años.

El niño, que se encuentra bien de salud, ha quedado a cargo de la madre y el caso está en manos del Grupo de Menores de la Policía Nacional de Talavera de la Reina.

\footnotetext{
${ }^{2}$ La noticia fue publicada en la versión digital de Las Provincias: http://www.lasprovincias.es/sucesos/rescatan-nino-anos-20170926103954-ntrc.html
} 